\title{
The nucleon-nucleon interaction up to sixth order in the chiral expansion
}

\author{
Ruprecht Machleidt* \\ University of Idaho, Moscow, Idaho, USA \\ E-mail: machleideuidaho.edu
}

\section{David R. Entem}

Universidad de Salamanca, Salamanca, Spain

E-mail: entemeusal.es

\section{Norbert Kaiser}

Technische Universitât München, Garching, Germany

E-mail: nkaisereph.tum.de

\section{Yevgen Nosyk}

University of Idaho, Moscow, Idaho, USA

E-mail: nosy6170@vandals.uidaho.edu

\begin{abstract}
We calculate the nucleon-nucleon $(N N)$ interaction up to sixth order $\left(\mathrm{N}^{5} \mathrm{LO}\right)$ of chiral perturbation theory. Previous studies extended only up to $\mathrm{N}^{3} \mathrm{LO}$ (fourth order) and typically showed a surplus of attraction, particularly, when the $\pi N$ LECs from $\pi N$ analysis were applied consistently. Furthermore, the contributions at $\mathrm{N}^{2} \mathrm{LO}$ and $\mathrm{N}^{3} \mathrm{LO}$ are both fairly sizeable, thus, raising concerns about the convergence of the chiral expansion. We show that the $\mathrm{N}^{4} \mathrm{LO}$ contribution is repulsive and, essentially, cancels the excessive attraction of $\mathrm{N}^{3} \mathrm{LO}$. The $\mathrm{N}^{5} \mathrm{LO}$ contribution turns out to be considerably smaller than the $\mathrm{N}^{4} \mathrm{LO}$ one, hence, establishing the desired trend of convergence. The predictions at $\mathrm{N}^{5} \mathrm{LO}$ are in excellent agreement with the empirical phase shifts of peripheral partial waves.
\end{abstract}

The 8th International Workshop on Chiral Dynamics

29 June 2015 - 03 July 2015

Pisa, Italy

\footnotetext{
* Speaker.
} 


\section{Introduction}

The derivation of nuclear forces from chiral effective field theory has been a topic of active research for the past quarter century (see Refs. [1,2] for recent reviews). By 1998, the evaluation of the nucleon-nucleon $(N N)$ interaction up to next-to-next-to-leading order $\left(\mathrm{N}^{2} \mathrm{LO}\right.$, third order in small momenta) was completed $[3,4,5]$ and, by 2003 , these calculations were extended to $\mathrm{N}^{3} \mathrm{LO}[6,7]$.

These $\mathrm{N}^{3} \mathrm{LO} N N$ potentials complemented by chiral three-nucleon forces (3NFs) have been applied in calculations of few-nucleon reactions, the structure of light- and medium-mass nuclei, and nuclear and neutron matter-with, in general, a good deal of success. However, some problems continue to exist that seem to defy any solution. The most prominent one is the so-called ' $A_{y}$ puzzle' of nucleon-deuteron scattering, which requires the inclusion of three-nucleon forces (3NFs). While the chiral $3 \mathrm{NF}$ at $\mathrm{N}^{2} \mathrm{LO}$ slightly improves the predictions for low-energy $N-d$ scattering [8], inclusion of the $\mathrm{N}^{3} \mathrm{LO} 3 \mathrm{NF}$ deteriorates the predictions [9]. Based upon general arguments, the $\mathrm{N}^{3} \mathrm{LO} 3 \mathrm{NF}$ is presumed weak, which is why one would not expect the solution of any substantial problems, anyhow. When working in the framework of an expansion, then, the obvious way to proceed is to turn to the next order, which is $\mathrm{N}^{4} \mathrm{LO}$ (or fifth order). Some $3 \mathrm{NF}$ topologies at $\mathrm{N}^{4} \mathrm{LO}$ have already been worked out $[10,11]$, and it has been shown that, at this order, all 22 possible isospin-spin-momentum 3NF structures appear. Moreover, the contributions are moderate to sizeable. What makes the fifth order even more interesting is the fact that, at this order, a new set of 3NF contact interactions appears, which has recently been derived by the Pisa group [12]. $3 \mathrm{NF}$ contact terms are of great interest from the point of view of the practitioner, because they are typically simple (as compared to loop contributions) and their coefficients are essentially free. Thus, at $\mathrm{N}^{4} \mathrm{LO}$, the $A_{y}$ puzzle may be solved in a trivial way through $3 \mathrm{NF}$ (contact) interactions. Due to the great diversity of structures offered at $\mathrm{N}^{4} \mathrm{LO}$, one can also expect that other persistent nuclear structure problems may finally find their solution at $\mathrm{N}^{4} \mathrm{LO}$.

A principle of all EFTs is that, for reliable predictions, it is necessary that all terms included are to be evaluated at the order at which the calculation is conducted. Thus, if nuclear structure problems require for their solution the inclusion of $3 \mathrm{NFs}$ at $\mathrm{N}^{4} \mathrm{LO}$, then also the two-nucleon force involved in the calculation has to be of order $\mathrm{N}^{4} \mathrm{LO}$. This is one reason why we investigate the $N N$ interaction at $\mathrm{N}^{4} \mathrm{LO}$. Besides this, there are also some more specific issues that motivate a study of this kind. From calculations of the $N N$ interaction at $\mathrm{N}^{2} \mathrm{LO}$ [4] and $\mathrm{N}^{3} \mathrm{LO}$ [6], it is wellknown that there is a problem with excessive attraction, particularly, when for the $c_{i}$ low-energy constants (LECs) of the dimension-two $\pi N$ Lagrangian the values are applied that are obtained from $\pi N$ analysis. It is important to know if this problem is finally solved when going beyond $\mathrm{N}^{3} \mathrm{LO}$. Last not least, also the convergence of the chiral expansion of the $N N$ interaction is of general interest.

In our calculations, we show that the $2 \pi$ - and $3 \pi$-exchange contributions at $\mathrm{N}^{4} \mathrm{LO}$ are prevailingly repulsive and, thus, are able to fully compensate the excessive attraction of the lower orders [13]. However, we also notice that the $\mathrm{N}^{2} \mathrm{LO}, \mathrm{N}^{3} \mathrm{LO}$, and $\mathrm{N}^{4} \mathrm{LO}$ contributions are all roughly of the same magnitude, raising legitimate concerns about the convergence of the chiral expansion of the $N N$-potential.

Therefore, we also investigate the $N N$-interaction at $\mathrm{N}^{5} \mathrm{LO}$ (sixth power in small momenta) with the goal to obtain more insight into the convergence issue. 
Besides this, the order $\mathrm{N}^{5} \mathrm{LO}$ has other interesting features. At this order, a new set of $N N$ contact terms depending with the sixth power on momenta appears, bringing the total number of short-distance parameters to 50. This set includes then terms that contribute up to $F$-waves.

However, the focus of our present contribution is on peripheral partial waves with orbital angular momentum $L \geq 4$, which are exclusively ruled by the non-polynomial pion-exchange expressions constrained by chiral symmetry. Hence, this investigation is a test of the implications of chiral symmetry for the $N N$-interaction up to sixth order.

\section{Pion-exchange contributions to the $N N$ potential at $\mathrm{N}^{4} \mathrm{LO}$ and $\mathrm{N}^{5} \mathrm{LO}$}

Contributions are derived in terms of their spectral functions, from which the momentumspace amplitudes $V_{\alpha}(q)$ are obtained via subtracted dispersion integrals. For fifth order $\left(\mathrm{N}^{4} \mathrm{LO}\right)$ contributions, the dispersion integrals read:

$$
\begin{aligned}
V_{C, S}(q) & =-\frac{2 q^{6}}{\pi} \int_{n m_{\pi}}^{\tilde{\Lambda}} d \mu \frac{\operatorname{Im} V_{C, S}(i \mu)}{\mu^{5}\left(\mu^{2}+q^{2}\right)}, \\
V_{T}(q) & =\frac{2 q^{4}}{\pi} \int_{n m_{\pi}}^{\tilde{\Lambda}} d \mu \frac{\operatorname{Im} V_{T}(i \mu)}{\mu^{3}\left(\mu^{2}+q^{2}\right)},
\end{aligned}
$$

and for sixth order, we use:

$$
\begin{aligned}
V_{C, S}(q) & =\frac{2 q^{8}}{\pi} \int_{n m_{\pi}}^{\tilde{\Lambda}} d \mu \frac{\operatorname{Im} V_{C, S}(i \mu)}{\mu^{7}\left(\mu^{2}+q^{2}\right)}, \\
V_{T}(q) & =-\frac{2 q^{6}}{\pi} \int_{n m_{\pi}}^{\tilde{\Lambda}} d \mu \frac{\operatorname{Im} V_{T}(i \mu)}{\mu^{5}\left(\mu^{2}+q^{2}\right)} .
\end{aligned}
$$

The subscripts $C, S$, and $T$ refer to central, spin-spin, and tensor force potentials, respectively. The thresholds are given by $n=2$ for two-pion exchange and $n=3$ for three-pion exchange. For $\tilde{\Lambda} \rightarrow \infty$, the above dispersion integrals yield the finite parts of loop-functions as in dimensional regularization, while for finite $\tilde{\Lambda}>>n m_{\pi}$, we have the method known as spectral-function regularization (SFR). The purpose of the finite scale $\tilde{\Lambda}$ is to constrain the imaginary parts to the low-momentum region where chiral effective field theory is applicable.

\subsection{Two-pion exchange contributions at $\mathrm{N}^{4} \mathrm{LO}$}

The $2 \pi$-exchange contributions that occur at $\mathrm{N}^{4} \mathrm{LO}$ are displayed graphically in Fig. 1. For part (a), the spectral functions are obtained by integrating the product of the leading one-loop $\pi N$ amplitude and the chiral $\pi \pi N N$ vertex proportional to $c_{i}$ over the Lorentz-invariant $2 \pi$-phase space. For part (b), the product of the one-loop $\pi N$ amplitude proportional to $c_{i}$ (see Ref. [10] for details) and the leading order chiral $\pi N$ amplitude are integrated over the $2 \pi$-phase space.

A few representative relativistic corrections are shown in Fig. 1(c). This group consists of diagrams with one vertex proportional to $c_{i}$ and one $1 / M_{N}$ correction, where $M_{N}$ denotes the nucleon mass. Since in this investigation we count $Q / M_{N} \sim\left(Q / \Lambda_{\chi}\right)^{2}$ (with $\Lambda_{\chi}$ denoting the chiral symmetry breaking scale), these relativistic corrections are formally of order $\mathrm{N}^{4} \mathrm{LO}$. 

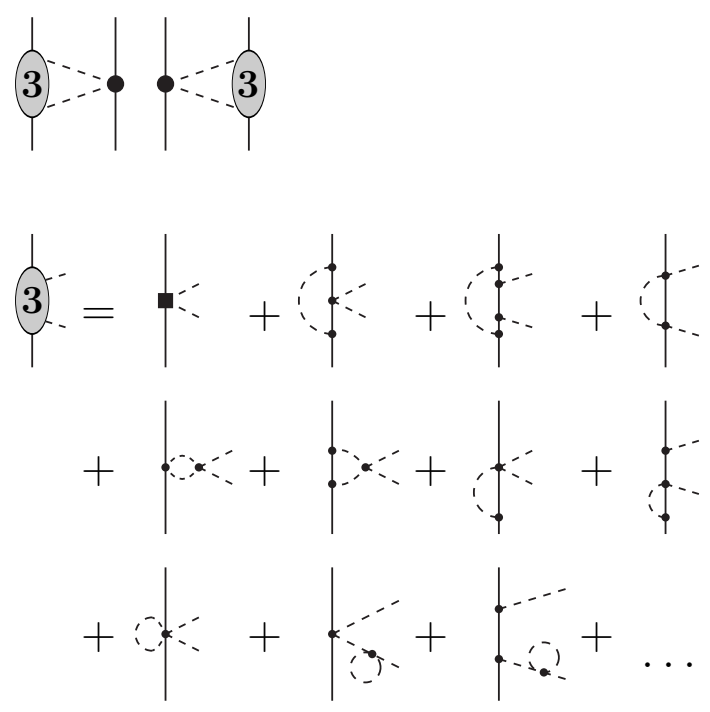

(a)
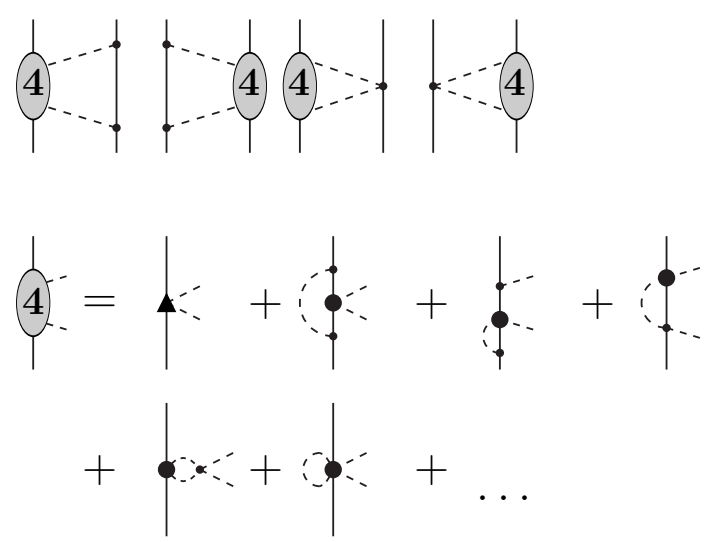

(b)

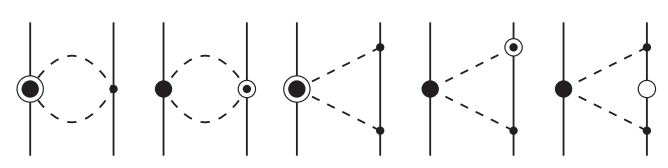

(c)

Figure 1: Two-pion-exchange contributions at $\mathrm{N}^{4} \mathrm{LO}$. (a) The leading one-loop $\pi N$ amplitude is folded with the chiral $\pi \pi N N$ vertices proportional to $c_{i}$. (b) The one-loop $\pi N$ amplitude proportional to $c_{i}$ is folded with the leading order chiral $\pi N$ amplitude. (c) Relativistic corrections of $N^{2} \mathrm{LO}$ diagrams. Solid lines represent nucleons and dashed lines pions. Small dots, large solid dots, solid squares, and triangles denote vertices of chiral order 1,2, 3 and 4, respectively. Shaded ovals represent complete $\pi N$-scattering amplitudes with their order specified by the number in the oval. Open circles are relativistic $1 / M_{N}$ corrections. 

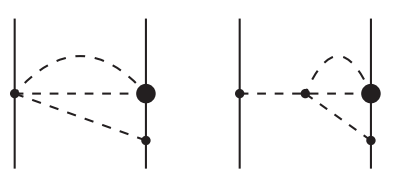

Class X

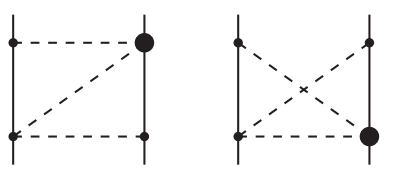

Class XII

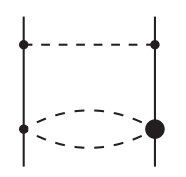

Class XI
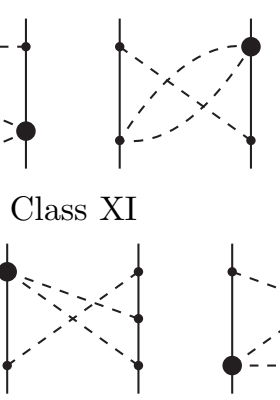

Class XIII

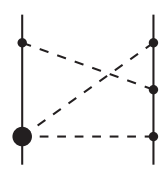

Class XIV

Figure 2: Three-pion exchange contributions at $\mathrm{N}^{4} \mathrm{LO}$. The classification scheme of Ref. [14] is used. Notation as in Fig. 1.

\subsection{Three-pion exchange contributions at $\mathrm{N}^{4} \mathrm{LO}$}

The $3 \pi$-exchange of order $\mathrm{N}^{4} \mathrm{LO}$ is shown in Fig. 2 . The spectral functions for these diagrams have been calculated in Ref. [14]. We use here the classification scheme introduced in that reference and note that class XI vanishes. Moreover, we find that class X and part of class XIV make only negligible contributions. Thus, we include in our calculations only class XII and XIII, and the $V_{S}$ contribution of class XIV.

\subsection{Two-pion exchange contributions at $\mathbf{N}^{5} \mathrm{LO}$}

The $2 \pi$-exchange contributions that occur at $\mathrm{N}^{5} \mathrm{LO}$ are displayed graphically in Fig. 3. For class (a), the spectral functions are obtained by integrating the product of the subleading one-loop $\pi N$-amplitude (see Ref. [10] for details) and the chiral $\pi \pi N N$-vertex proportional to $c_{i}$ over the Lorentz-invariant $2 \pi$-phase space.

A first set of $2 \pi$-exchange contributions at three-loop order, denoted by class (b), is displayed in Fig. 3(b). For this class of diagrams, the leading one-loop $\pi N$-scattering amplitude is multiplied with itself and integrated over the $2 \pi$-phase space.

Further $2 \pi$-exchange three-loop contributions at $\mathrm{N}^{5} \mathrm{LO}$, denoted by class (c), are shown in Fig. 3(c). For these the two-loop $\pi N$-scattering amplitude (which is of order five) would have to be folded with the tree-level $\pi N$-amplitude. To our knowledge, the two-loop elastic $\pi N$-scattering amplitude has never been evaluated in some decent analytical form. Note that the loops involved in the class (c) contributions include only leading order chiral $\pi N$-vertices. According to our experience such contributions are typically small. For these reasons we omit class (c) in the present calculation.

\subsection{Three-pion exchange contributions at $\mathrm{N}^{5} \mathrm{LO}$}

The $3 \pi$-exchange contributions of order $\mathrm{N}^{5} \mathrm{LO}$ are shown in Fig. 4. The diagrams displayed in Fig. 4(a) are characterized by the presence of a subleading $\pi \pi N N$-vertex in each nucleon line. Using a notation introduced in Refs. [13, 14], we distinguish between the various sub-classes of diagrams by roman numerals. In Fig. 4(b), each $3 \pi$-exchange diagram includes the one-loop $\pi N$ amplitude (completed by the low-energy constants $\bar{d}_{j}$ ). These contributions are, in general, small. 


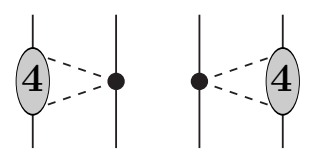

(a)

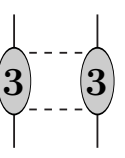

(b)

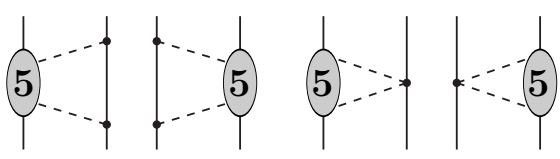

(c)

Figure 3: Two-pion-exchange contributions to the $N N$-interaction at $\mathrm{N}^{5} \mathrm{LO}$. (a) The subleading one-loop $\pi N$-amplitude is folded with the chiral $\pi \pi N N$-vertices proportional to $c_{i}$. (b) The leading one-loop $\pi N$ amplitude is folded with itself. (c) The leading two-loop $\pi N$-amplitude is folded with the tree-level $\pi N$ amplitude. Notation as in Fig. 1.

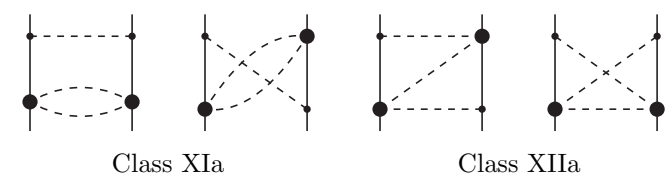

(a)

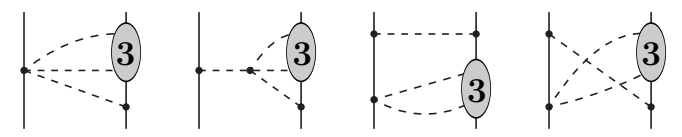

Class Xb Class XIb
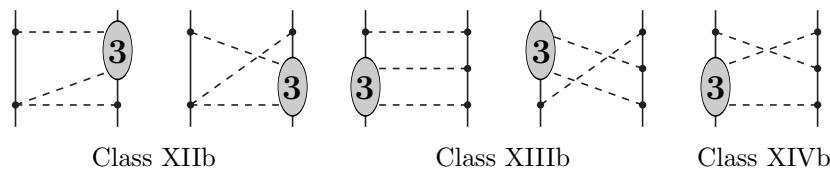

(b)

Figure 4: Three-pion exchange contributions at $\mathrm{N}^{5} \mathrm{LO}$. (a) Diagrams proportional to $c_{i}^{2}$. (b) Diagrams involving the one-loop $\pi N$-amplitude. Roman numerals refer to sub-classes following the scheme introduced in Refs. [13, 14]. Notation as in Fig. 1. 


\subsection{Four-pion exchange at $\mathrm{N}^{5} \mathrm{LO}$}

The exchange of four pions between two nucleons occurs for the first time at $\mathrm{N}^{5} \mathrm{LO}$. The pertinent diagrams involve three loops and only leading order vertices, which explains the sixth power in small momenta. Three-pion exchange with just leading order vertices is known to be negligibly small, and so we expect four-pion exchange with leading order vertices to be even smaller. Therefore, we safely neglect this contribution.

\section{Perturbative $N N$-scattering in peripheral partial waves}

To obtain an idea of the physical relevance and implications of the contributions evaluated in Sec. 2, we will now calculate the impact of these on elastic $N N$-scattering in peripheral partial waves. Specifically, we will consider partial waves with orbital angular momentum $L \geq 4$ (i.e., $G$ waves and higher), because polynomial terms up to sixth power do not make any contributions to these angular momentum states. The $L \geq 4$ partial waves are sensitive only to the non-polynomial pion-exchange expressions governed by chiral symmetry.

The smallness of the phase-shifts in peripheral partial waves suggests that the calculation can be done perturbatively. This avoids the complications and possible model-dependences (e.g., cutoff-dependence) that the non-perturbative treatment with the Lippmann-Schwinger equation, necessary for low partial waves, is beset with.

Chiral symmetry establishes a link between the dynamics in the $\pi N$-system and the $N N$-system (through common low-energy constants). In order to check the consistency, we use the LECs for subleading $\pi N$-couplings as determined in analyses of low-energy elastic $\pi N$-scattering. Appropriate analyses for our purposes are contained in Refs. [10, 15], where $\pi N$-scattering has been calculated at fourth order using the same power-counting of relativistic $1 / M_{N}$-corrections as in the present work. Ref. [10] performed two fits, one to the GW [16] and one to the KH [17] partial wave analysis. In our present work, we apply the GW set. Moreover, we absorb the Goldberger-Treiman discrepancy into an effective value of the nucleon axial-vector coupling constant $g_{A}=g_{\pi N N} f_{\pi} / M_{N}=1.29$.

The predictions for all $G$ and $H$ waves, are displayed in Fig. 5 in terms of colored bands that are generated by varying the SFR cutoff $\tilde{\Lambda}$ between 700 and $900 \mathrm{MeV}$. The figure clearly reveals that, at $\mathrm{N}^{3} \mathrm{LO}$, the predictions are, in general, too attractive. The $\mathrm{N}^{4} \mathrm{LO}$ contribution, essentially, compensates this attractive surplus. And turning to the result at $\mathrm{N}^{5} \mathrm{LO}$ : it shows a moderate repulsive contribution bringing the final prediction right onto the data (i.e. empirical phase-shifts). Moreover, the $\mathrm{N}^{5} \mathrm{LO}$ contribution is, in general, substantially smaller than the one at $\mathrm{N}^{4} \mathrm{LO}$, thus, establishing a clear signature of convergence of the chiral expansion.

Figure 5 includes only the three highest orders. However, a comparison between all orders is also of interest. Therefore, we show in Figs. 6 the contributions to phase shifts through all six chiral orders from LO to $\mathrm{N}^{5} \mathrm{LO}$. Note that the difference between the LO prediction (one-pionexchange, dotted line) and the data (filled and open circles) is to be provided by two- and three-pion exchanges, i.e. the intermediate-range part of the nuclear force. How well that is accomplished is a crucial test for any theory of nuclear forces. NLO produces only a small contribution, but $\mathrm{N}^{2} \mathrm{LO}$ creates substantial intermediate-range attraction (most clearly seen in ${ }^{1} G_{4}$ and ${ }^{3} G_{5}$ ). In 

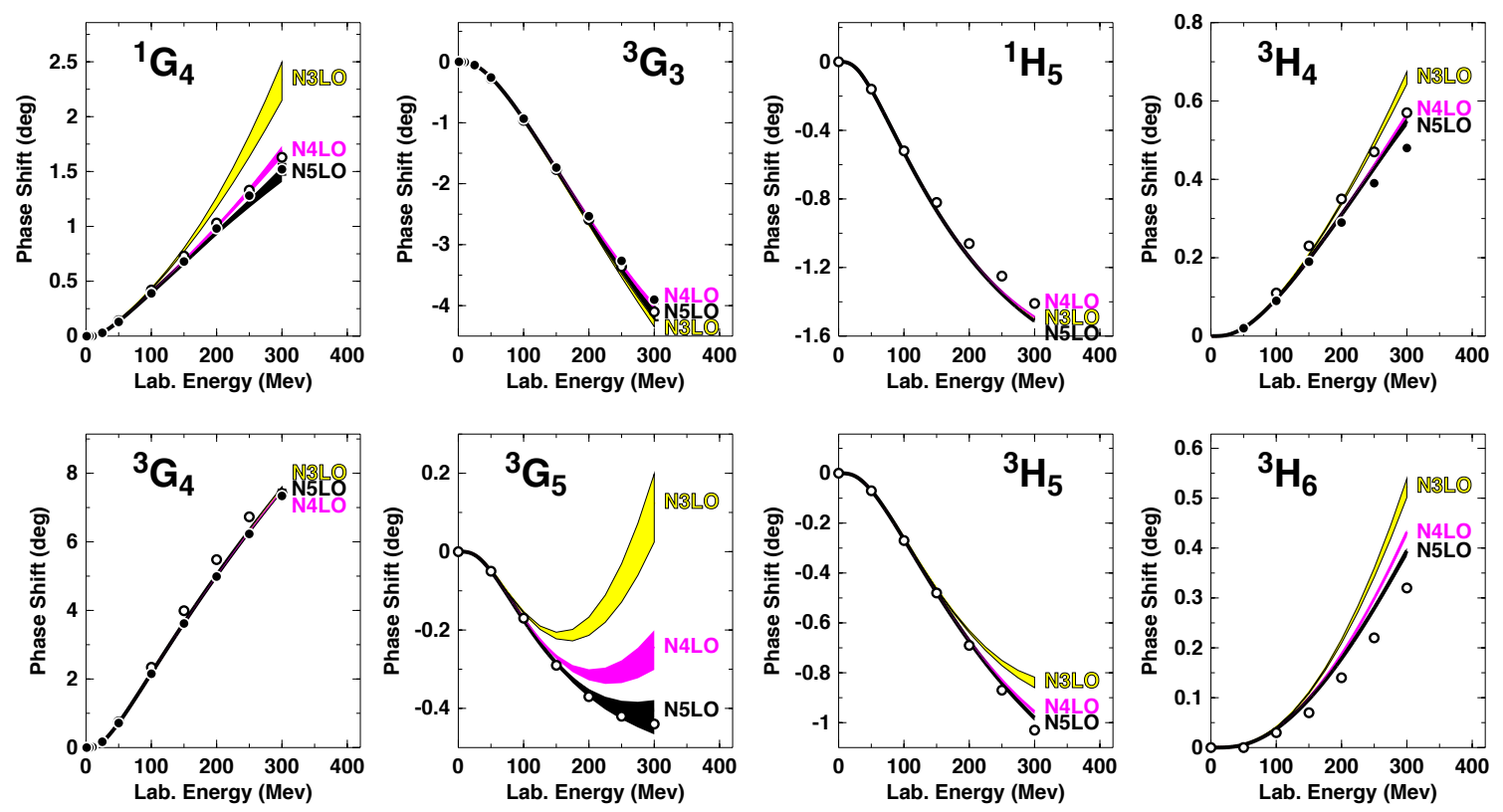

Figure 5: Phase-shifts of neutron-proton scattering in $G$ and $H$ waves at various orders as denoted. The colored bands show the variations of the predictions when the SFR cutoff $\tilde{\Lambda}$ is changed over the range 700 to $900 \mathrm{MeV}$. The filled and open circles represent the results from the Nijmegen multi-energy $n p$ phase-shift analysis [18] and the GWU SP07 $n p$-analysis [19], respectively.
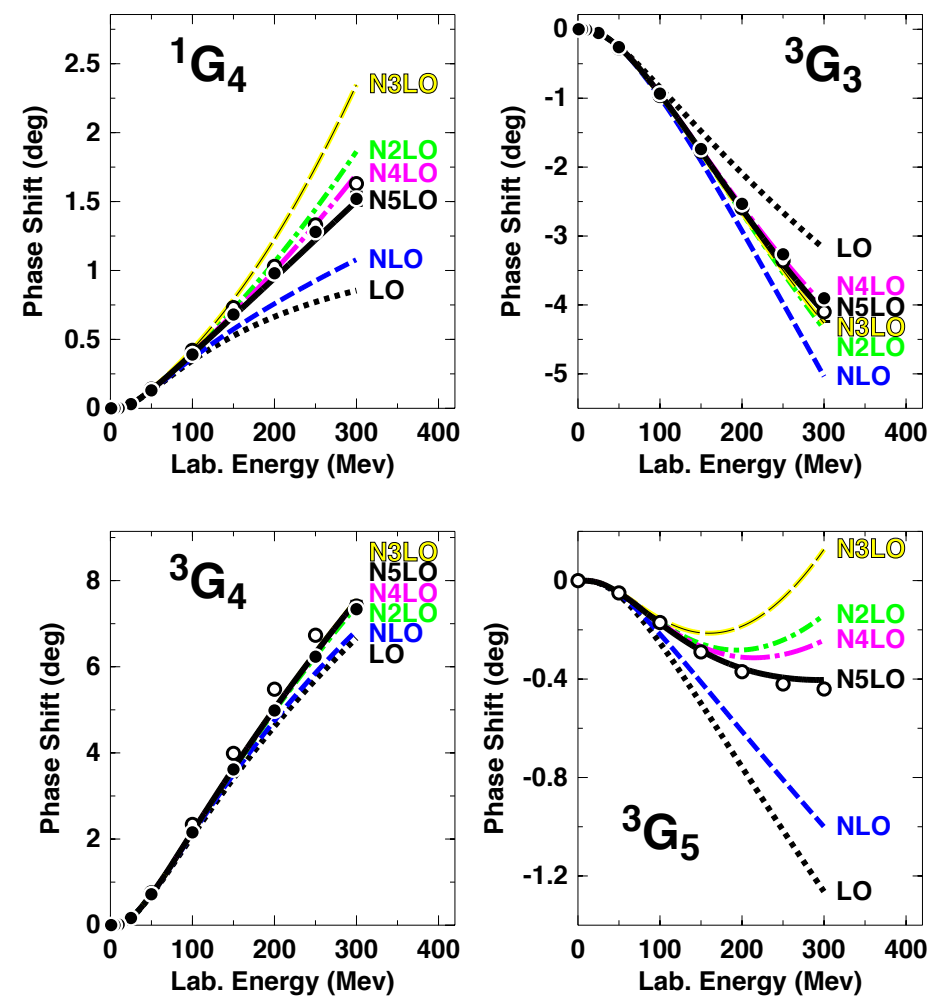

Figure 6: Phase-shifts of neutron-proton scattering in $G$ waves at all orders from $\mathrm{LO}$ to $\mathrm{N}^{5} \mathrm{LO}$. A SFR cutoff $\tilde{\Lambda}=800 \mathrm{MeV}$ is used. Empirical phase shifts are as in Fig. 5 . 
fact, $\mathrm{N}^{2} \mathrm{LO}$ is the largest contribution among all orders. This is due to the one-loop $2 \pi$-exchange (2PE) triangle diagram which involves one $\pi \pi N N$-contact vertex proportional to $c_{i}$. This vertex represents correlated 2PE as well as intermediate $\Delta(1232)$-isobar excitation. It is well-known from the traditional meson theory of nuclear forces that these two features are crucial for a realistic and quantitative $2 \mathrm{PE}$ model. Consequently, the one-loop $2 \mathrm{PE}$ at $\mathrm{N}^{2} \mathrm{LO}$ is attractive and assumes a realistic size describing the intermediate-range attraction of the nuclear force about right. At $\mathrm{N}^{3} \mathrm{LO}$, more one-loop 2PE is added by the bubble diagram with two $c_{i}$-vertices, a contribution that seemingly is overestimating the attraction. This attractive surplus is then compensated by the prevailingly repulsive two-loop $2 \pi$ - and $3 \pi$-exchanges that occur at $\mathrm{N}^{4} \mathrm{LO}$ and $\mathrm{N}^{5} \mathrm{LO}$.

\section{Conclusions}

In this contribution, we have calculated $2 \pi$ - and $3 \pi$-exchange contributions to the $N N$-interaction which occur at $\mathrm{N}^{4} \mathrm{LO}$ (fifth order) and $\mathrm{N}^{5} \mathrm{LO}$ (sixth order) of the chiral low-momentum expansion. The calculations are done in heavy-baryon chiral perturbation theory using the most general fourth order Lagrangian for pions and nucleons. We apply low-energy constants for subleading $\pi N$ coupling, which were determined from an analysis of elastic $\pi N$-scattering to fourth order using the same power counting scheme as in the present work. The spectral functions, which determine the $N N$-amplitudes via subtracted dispersion integrals, are regularized by a cutoff $\tilde{\Lambda}$ in the range 0.7 to $0.9 \mathrm{GeV}$. Besides the cutoff $\tilde{\Lambda}$, our calculations do not involve any adjustable parameters.

From past work on $N N$ scattering in chiral perturbation theory (see, e.g., Ref. [6]), it is wellknown that, at $\mathrm{N}^{2} \mathrm{LO}$ and $\mathrm{N}^{3} \mathrm{LO}$, chiral $2 \pi$-exchange produces far too much attraction. One important result of the present study is that the $\mathrm{N}^{4} \mathrm{LO} 2 \pi$ and $3 \pi$ contributions are prevailingly repulsive and, thus, compensate the excessive attraction of the lower orders.

On the other hand, the $\mathrm{N}^{2} \mathrm{LO}, \mathrm{N}^{3} \mathrm{LO}$, and $\mathrm{N}^{4} \mathrm{LO}$ contributions are all about of the same magnitude raising some concern about the convergence of the chiral expansion of the $N N$ amplitude. To obtain insight into this issue, we have also investigated the $N N$ interaction at $\mathrm{N}^{5} \mathrm{LO}$. Our calculations show that the contribution at $\mathrm{N}^{5} \mathrm{LO}$ is substantially smaller than the one at $\mathrm{N}^{4} \mathrm{LO}$, thus, establishing a clear signature of convergence. The $\mathrm{N}^{5} \mathrm{LO}$ two-loop $2 \pi$-exchange contribution is the largest, while the corresponding three-loop contribution is small, but not negligible. Three-pion exchange is generally small at $\mathrm{N}^{5} \mathrm{LO}$. The phase-shift predictions in $G$ and $H$ waves, where only the non-polynomial terms governed by chiral symmetry contribute, are in excellent agreement with the data.

This investigation represents the most comprehensive (and successful) test of the implications of chiral symmetry for the $N N$-system.

\section{Acknowledgements}

This work was supported in part by the U.S. Department of Energy under Grant No. DE-FG0203ER41270 (R.M. and Y.N.), the Ministerio de Ciencia y Tecnología under Contract No. FPA201347443-C2-2-P and the European Community-Research Infrastructure Integrating Activity "Study of Strongly Interacting Matter" (HadronPhysics3 Grant No. 283286) (D.R.E.), and by DFG and NSFC (CRC110) (N.K.). 


\section{References}

[1] R. Machleidt and D. R. Entem, Chiral effective field theory and nucler forces, Phys. Rep. 503 (2011) 1 .

[2] E. Epelbaum, H.-W. Hammer, and U.-G. Meißner, Modern theory of nuclear forces, Rev. Mod. Phys. 81 (2009) 1773.

[3] C. Ordóñez, L. Ray, and U. van Kolck, Two-nucleon potential from chiral Lagrangians, Phys. Rev. C 53 (1996) 2086.

[4] N. Kaiser, R. Brockmann, and W. Weise, Peripheral nucleon-nucleon phase shifts and chiral symmetry, Nucl. Phys. A625 (1997) 758.

[5] N. Kaiser, S. Gerstendörfer, and W. Weise, Peripheral NN-scattering: role of delta-excitation, correlated two-pion and vector meson exchange, Nucl. Phys. A637 (1998) 395.

[6] D. R. Entem and R. Machleidt, Chiral $2 \pi$ exchange at fourth order and peripheral $N N$ scattering, Phys. Rev. C 66 (2002) 014002.

[7] D. R. Entem and R. Machleidt, Accurate charge-dependent nucleon-nucleon potential at fourth order of chiral perturbation theory, Phys. Rev. C 68 (2003) 041001.

[8] M. Viviani, L. Girlanda, A. Kievsky, and L. E. Marcucci, Effect of three-nucleon interactions in $p-{ }^{3} H e$ elastic scattering, Phys. Rev. Lett. 111 (2013) 172302.

[9] J. Golak et al., Low-energy neutron-deuteron reactions with $N^{3}$ LO chiral forces, arXiv:1410.0756 [nucl-th].

[10] H. Krebs, A. Gasparyan, and E. Epelbaum, Chiral three-nucleon forces at $N^{4} L O$ : Longest-range contributions, Phys. Rev. C 85 (2012) 054006.

[11] H. Krebs, A. Gasparyan, and E. Epelbaum, Chiral three-nucleon forces at $N^{4} L O$ : Intermediate-range contributions, Phys. Rev. C 87 (2013) 054007.

[12] L. Girlanda, A. Kievsky, M. Viviani, Subleading contributions to the three-nucleon contact interaction, Phys. Rev. C 84 (2011) 014001.

[13] D. R. Entem, N. Kaiser, R. Machleidt, and Y. Nosyk, Peripheral nucleon-nucleon scattering at fifth order of chiral perturbation theory, Phys. Rev. C 91 (2015) 014002

[14] N. Kaiser, Chiral $3 \pi$-exchange NN potentials: Results for dominant next-to-leading-order contributions, Phys. Rev. C 63 (2001) 044010.

[15] K. A. Wendt, B. D. Carlsson, and A. Ekstrőm, Uncertainty Quantification of the Pion-Nucleon Low-Energy Coupling Constants up to Fourth Order in Chiral Perturbation Theory, arXiv:1410.0646 [nucl-th].

[16] R. A. Arndt, W. J. Briscoe, I. I. Strakovsky, and R. L. Workman, Extended partial-wave analysis of $\pi N$ scattering data, Phys. Rev. C 74 (2006) 045205.

[17] R. Koch, Improved $\pi N$ partial waves consistent with analyticity and unitarity, Nucl. Phys. A 448 (1986) 707.

[18] V. G. J. Stoks, R. A. M. Klomp, M. C. M. Rentmeester, and J. J. de Swart, Partial-wave analysis of all nucleon-nucleon scattering data below 350 MeV, Phys. Rev. C 48 (1993) 792.

[19] W. J. Briscoe, I. I. Strakovsky, and R. L. Workman, SAID Partial-Wave Analysis Facility, Data Analysis Center, The George Washington University, solution SP07 (Spring 2007). 